\title{
Patients experience of viewing time-lapse sequences: A prospective survey study.
}

\author{
Annika Bladh Blomquis ${ }^{1}$, Raghad Samir ${ }^{2}$, Ann-Britt Engström ${ }^{2}$, Staffan Nilsson $^{2}$, Anna-Karin Lind ${ }^{3}$, \\ Göran Westlander ${ }^{3}$, Sophie Larsson ${ }^{3}$, Thorir Hardarson ${ }^{3}$, Julius Hreinsson ${ }^{4 *}$ \\ ${ }^{1}$ Nordic IVF Center, Odinsgatan 10, Gothenburg, SE 411 03, Sweden \\ ${ }^{2}$ IVF-Sweden, IVF-clinic Falun, Falun, SE 791 71, Sweden \\ ${ }^{3}$ IVF-Sweden, Fertility Centre Gothenburg, Gothenburg, SE 402 29, Sweden \\ ${ }^{4}$ Department of Immunology, Genetics and Pathology, Rudbeck Laboratory, Uppsala, SE 751 85, Sweden
}

\begin{abstract}
The use of time-lapse monitoring in ART is spreading rapidly and is already considered an important part of treatment in many centers internationally, but studies are lacking on patients experience of using this technology. We consider it important not to forget patient-oriented aspects of new technologies. The study question was therefore; how do patients value the chance to view time-lapse sequences of their embryos at the time of embryo transfer?

We performed a prospective, observational questionnaire study at three of IVF Sweden's centers, including 242 patients. We report the results from self-evaluated scores of patient's experience which were reported anonymously. Participants in the study were patients who were offered to view time-lapse sequences of embryos at the time of embryo transfer. A 5-grade Likert-scale instrument was used for assessing patient's experience.

A majority of the patients found viewing the sequences to be a relevant and important aspect of their treatments. Patients felt increased participation in, and increased satisfaction with the treatment. There was a clear interest in obtaining a copy of the time-lapse sequence but only if the treatment resulted in a pregnancy.

If time-lapse technology is available in a clinic it could be an additive value to share the sequences with the patients as deepened information about their treatment. If a copy of the sequences is made available to the patients we recommend to delay the time for that at least until there is a verified ongoing pregnancy.
\end{abstract}

Keywords: Embryo transfer, Fertilization in vitro, Time-lapse, Patient satisfaction.

Accepted on December 21, 2016

\section{Introduction}

Continuous monitoring of embryo development with time-lapse technology has been utilized in assisted reproduction for several years, both for research and in recent years in clinical practice. This entails a combination of digital photography, incubation and culture, high-magnification microscopy and computer processing of digital images to form short time-lapse sequences. The embryo is photographed at 10-20 minute intervals without the need of removing it from the incubator for this purpose. The information obtained through this technology has been important in increasing the understanding of the development of human pre-implantation embryos in vitro [1,2]. During the past 5-6 years, a rapid increase in the production and availability of this technology has led to its large-scale use in assisted reproduction [3].

A number of studies have been published where the effects of the use of time-lapse technology and its application in assisted reproduction treatments has been studied $[4,5]$.

Focus has been on validating evaluation parameters of embryo development to maximize utilization of the technology. Many of these studies show a positive effect with increase in pregnancy rates as a consequence of using these new evaluation parameters. Recently a prospective randomized study shows clinical advantages in reproductive outcomes when using timelapse technology [6]. Concurrent with these new developments, a number of IVF-clinics have chosen to make the information included in the time-lapse sequences accessible for their patients in the form of video-sequences showing the details of the development of their embryos. According to available information, the experience of this new practice is satisfying although studies are lacking on how patients experience this new source of information $[7,8]$.

Implementation of this new technology can be compared with the advent of ultrasound imaging during pregnancy in the late 1960's. The diagnostic value of ultrasound imaging was acknowledged early on whereas the impact on patient's experience of pregnancy and potential negative psychological effects were debated [9]. With technological improvements and general use of ultrasound technology, it has become clear however that patients in general are positive and welcome the opportunity for potential bonding with the child-to-be [10,11].

Ethical aspects of innovative technology in reproductive- and maternity care need scrutiny to make sure those patient aspects are not forgotten in the ever-increasing speed of technological development. We wish to add the patient perspective to the ongoing debate on the potential benefits of the technology. 
Previous research indicates that patients have great expectations regarding their IVF-treatments. Stress level is high as well as concern regarding the possibilities to be able to become parents $[12,13]$.

It is conceivable that viewing the sequences of embryo development may create expectations which the treatment cannot meet if pregnancy is not achieved or if the pregnancy is prematurely terminated in cases of miscarriage. In case of a negative result of the treatment it might be possible that new questions are raised about the time-lapse evaluation. Some patients might want to reevaluate the sequences or have a second opinion in light of the result. It is important to seriously consider these questions and study how patients experience the implementation of new technologies.

The objective of this study was therefore to examine patient's experience of viewing time-lapse sequences of the development of the selected embryo/embryos at the time of embryo transfer.

\section{Material and Methods}

This was an anonymous survey study including patients at three privately funded IVF-clinics in Sweden.

The study is descriptive in its approach since it aims at describing an existing phenomenon although a quantitative method for data collection was used. Only primary data were collected. The three clinics included in the study were: IVF-kliniken CuraÖresund, Malmö; Fertilitetscentrum Gothenburg; IVF-kliniken Falun. All clinics are private enterprises and a part of the IVF-Sverige consortium. The time-lapse systems utilized were Primo Vision ${ }^{\circledR}$ (Malmö and Gothenburg) and EmbryoScope ${ }^{\circledR}$ (Falun). Both are produced and distributed by Vitrolife ${ }^{\circledR}$.

Most patients in these three clinics fund their own treatment costs and only a minority of the patients received state-funded treatments. The study did not collect data on that aspect and time-lapse culture did not entail any additional costs for patients.

No remuneration was offered to the patients for participation in the study. Patients having embryos cultured in the time-lapse systems during the study period were offered to view and to have their time-lapse sequences explained to them by an embryologist and/or a clinician The only exclusion criteria were inability to read Swedish as translation of the questionnaire form was not available. See Figure 1 for further details on patient recruitment.

The sequence was viewed at the time of embryo transfer and was explained as additional information to the regular description of the stage of embryo development at transfer. This additional information took approximately 5 minutes and included visualizing cell cleavages, appearance/disappearance of nuclei, dynamics of embryo development and possible fragmentation as well as explaining progression and frequency of cell cleavages. A vast majority of the transfers were single embryo transfers. If a double embryo transfer was performed, the time-lapse sequences of both embryos were shown to the patients. All patients who had embryos cultured under timelapse conditions were willing to view the sequences at the time of embryo transfer.

The time-lapse sequences were shown on a computer screen or tablet computer by the clinician performing the embryo transfer and/or by an embryologist. In case the patients requested, or if the clinician/embryologist wanted to review details, the sequence could be stopped and/or repeated. In all cases the same kind of information was given regarding embryo development and progression of cell divisions.

No selection of patient's was performed regarding embryo quality at the day of embryo transfer. Patients were recruited consecutively and only according to time-lapse culture availability, meaning that patients with varying embryo quality participated in the study. Because of the anonymous nature of the data collection, no correlation with embryo quality was possible.

In connection with the embryo transfer the clinician asked the couple to fill in the questionnaire form, details of which are given in Table 1. A great majority of the patients viewing the time-lapse sequences participated in the survey but we did not register if they did or not, since the questionnaire forms were left anonymously. Men and women completed separate survey forms. Patients were recruited consecutively in the participating clinics during the study period.

The study used a 5-grade Likert-scale instrument for assessing patient's experience. See Table 1 for details regarding the questions.

The alternatives for answering the Likert-scale questions were:

\section{1: Agree completely}

2: Mostly agree

\section{3: Neither}

\section{4: Mostly disagree}

\section{5: Completely disagree}

Demographic data and data on previous attempts were collected. If the couple both attended the transfer, they were each asked for participation, otherwise only the woman coming for embryo replacement participated in the study.

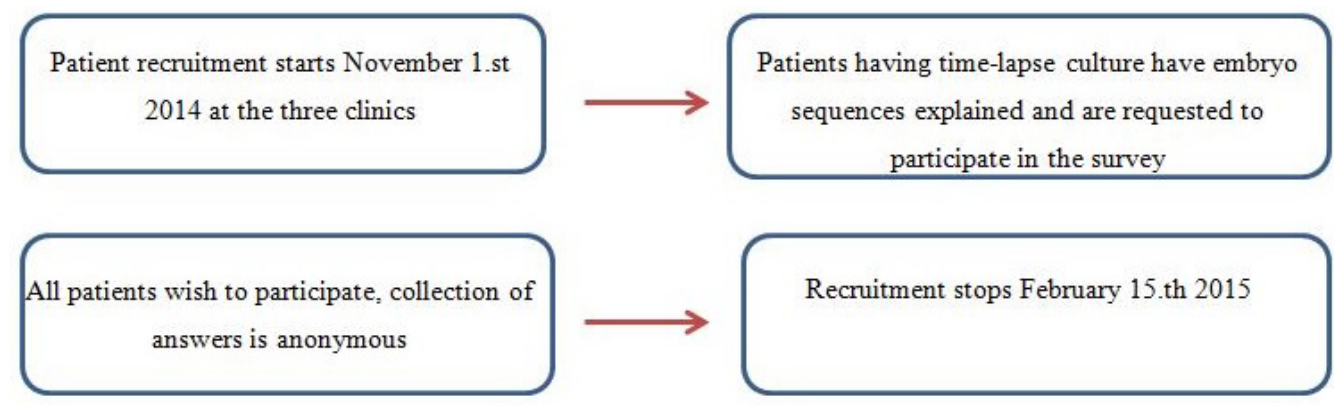

Figure 1. Flow chart of patient recruitment for the study 


\section{Data analysis}

Data was analyzed using an SPSS statistical processor (IBM, version 21) in order to run correlation- and descriptive analyses. The results were then presented in graphical forms as appropriate with further contextual interpretation. The Kruskal-Wallis significance test was performed for Likert scale questions.

\section{Limitations}

The number of recruited patients varied somewhat between the clinics but no obvious differences were observed. Some of the patients had state-funded treatment which may have affected the answers to questions regarding costs of the additional service in connection with time-lapse culture.

The sample studied here may to some degree be considered an opportunity sample since couples eligible to participate were those who had time-lapse culture of their embryos. This may limit the possible generalization of the results presented here to all IVF-patients. There was however no a-priory selection of cases or couples for time-lapse culture and all these couples were included during the study period so this limitation should not be a serious one. No drop-outs were observed; on the contrary patients were eager to participate and we consider the sample studied to be representative of the general population of patients coming for IVF-treatment.

The reliability and validity of the questionnaire were not tested beforehand and as this is the first study to evaluate patient's experience of viewing time-lapse sequences we consider this a pilot study in the field.

\section{Ethical considerations}

Application for ethical approval was sent to the regional ethics committee at Lund University. The committee considered the study to lie outside the boundaries of ethical vetting because of the non-invasive and anonymous nature of the study.

\section{Results}

The study included 242 respondents with an average age of 36 years. Of these $54 \%$ were female, $42 \%$ male, $4 \%$ did not answer that question.

The distribution of patients between clinics was: CuraOresund Malmö 26\%; Fertilitetscentrum Gothenburg 23\%; IVF-kliniken Falun 51\%. Average number of previous embryo transfers was 2.2. The average scores to questions 1 through 9 are shown graphically in Figure 2. Because of the non-parametric nature of the data, the mean, median and mode for the participant's responses are given in Table 2. Results of the correlation analysis regarding age, sex of the respondent, number of previous transfers as well as the clinic in question are also given in Table 2.

Patients expressed that viewing the time-lapse sequences gave them important and relevant information regarding their treatment. Furthermore, those who had previously had conventional oral and screen-viewing information found that less satisfactory compared with time-lapse viewing; however, the number of previous embryo transfers showed significant negative correlation with how important patients considered receiving information on embryo development in the laboratory

Table 1. Details of the questionnaire used in the study.

Categorical variable: Sex

Continuous variables: Age and number of previous attempts

Likert scale questions:

The sequence I viewed at the time of embryo transfer gave important and relevant information regarding our treatment.

In my experience of previous embryo transfers, oral information regarding the transferred embryo and/or viewing the embryo on screen at the time of transfer gave me adequate information.

It is important for me to know exactly how our embryos developed in the laboratory.

Seeing the embryo development increases my feeling of participation in our treatment.

I would prefer not to know too much about our embryo development.

I would like to have access to a copy of the time-lapse sequence of the transferred embryo for private use. (If not, please skip question \#7).

I would like to have access of such a copy, even if the treatment does not lead to a pregnancy.

I would consider paying extra for use of time-lapse technology in future treatment cycles.

Access to the time-lapse sequence would increase my satisfaction with the IVF-treatment.

Table 2. Mean, median and mode for all responses to the Likert-scale questions and results from the correlation analysis. n.s: not significant

\begin{tabular}{|c|c|c|c|c|c|c|c|}
\hline Question & Mean & Median & Mode & Sex & Age & $\begin{array}{l}\text { Previous } \\
\text { attempts }\end{array}$ & Clinic \\
\hline 1. The sequence gave relevant information & 4,5 & 5 & 5 & n.s. & n.s. & n.s. & n.s. \\
\hline 2. Oral information was adequate & 3,4 & 3 & 3 & n.s. & n.s. & n.s. & n.s. \\
\hline 3. Knowledge is important & 4,4 & 5 & 5 & n.s. & n.s. & $P=0.009$ & $p=0.006$ \\
\hline 4. Increased feeling of participation & 4,8 & 5 & 5 & n.s. & n.s. & n.s. & n.s. \\
\hline 5. Prefer not to know & 1,1 & 1 & 1 & n.s. & $P=0.029$ & n.s. & n.s. \\
\hline 6. Access to copy of the sequence & 3,9 & 5 & 5 & n.s. & n.s. & $P=0.036$ & $P=0.003$ \\
\hline 7. Access to copy even if no pregnancy & 2,4 & 2 & 1 & n.s. & n.s. & n.s. & n.s. \\
\hline 8. Would consider paying extra & 3,2 & 3 & 3 & n.s. & n.s. & n.s. & n.s. \\
\hline $\begin{array}{l}\text { 9. Access to sequence would increase } \\
\text { satisfaction }\end{array}$ & 3,7 & 4 & 5 & n.s. & n.s. & n.s. & n.s. \\
\hline
\end{tabular}




\section{Scores on Likert scale questions}

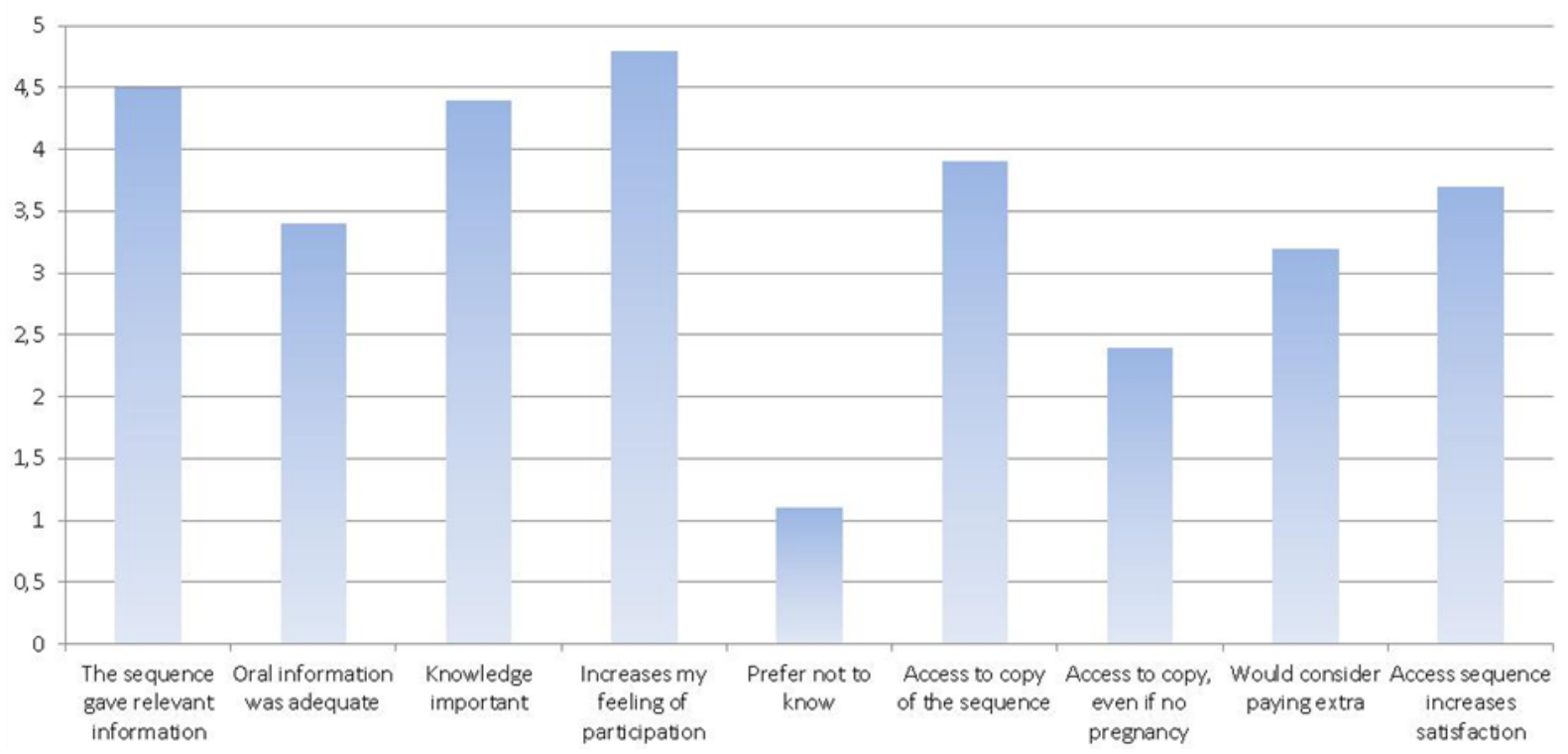

Figure 2. Average scores on Likert scale questions according to Table 1.

(Pearson correlation, 2-tailed, $\mathrm{p} \leq 0.009$ ). The number of previous transfers showed significant positive correlation with their desire to have access to the time-lapse sequence $(p=0.036)$. Patients expressed the opinion that the information on embryo development is important and that it increased their feeling of participation in the treatment. Patients do not wish to stay ignorant on the specifics of embryo development in the laboratory and there is a clear interest in having access to a copy of the time-lapse sequence. However, in case of a negative pregnancy test, the respondents were much less interested in having a copy of the sequence.

There was not much interest in paying extra for time-lapse culture and access to the sequences. Additional comments offered by patients in writing generally showed a positive response to viewing the time-lapse sequences although a few patients did not consider this an important aspect of their treatment.

Age seems to correlate with patients not preferring to know details of embryo development (Q 5) meaning that older patients are less interested in knowing details of embryo development by time-lapse technology. It is also clear from the complete correlation analysis that there is very good internal consistency in the data such that there is for example a positive correlation between finding the time-lapse sequences important, wishing to obtain a copy of them and to be willing to pay for time-lapse culture.

\section{Discussion}

This study is, to our knowledge, the first to systematically assess patients' experiences of viewing time lapse sequences showing embryo development. Clinicians and embryologists alike have the primary goal of increasing the efficacy of the treatments offered but sometimes the patients' perspective might not be sufficiently well considered. Assisted reproduction treatments are associated with emotional strain and high expectations from patients whereas overall success rates per treatment remain relatively low. In 2010 the clinical pregnancy rate per transfer only reached $32-33 \%$ according to data collected by ESHRE [14,15].
When the majority of treatment cycles do not result in a pregnancy, it is important to simultaneously improve results while also facilitating patients' coping strategies.

Research has shown that couples undergoing IVF-treatment are prone to seeing the pre-implantation embryo as their child and that professionals as a rule have a tendency to take a more pragmatic or distanced viewpoint [16]. In this context, it is reasonable that viewing time-lapse sequences could enhance these feelings and increase the emotional tie between the couple and the embryo. This is important to consider and may explain why couples in the study were reluctant to express a wish for a copy of the sequence unless a pregnancy had ensued.

Time-lapse imaging is a technique which in many ways can be compared to the use of fetal ultrasound. As described previously [9], fetal ultrasound is used as a technical diagnostic tool from a professional view, parents undergoing the examination however tend to see it as an experience that enhances the emotional bonding to the fetus. Ultrasound is in this aspect labeled as an "instrument of parenting" - the same could perhaps be applied for time-lapse. As is the case with time-lapse, not much academic attention is given today to the patient's experience of the examination.

As previous studies on fetal ultrasound show, the parallel to time-lapse is partly obvious - time-lapse is from a professional point of view primarily a technical method to improve the culture and the selection of embryos. However when sharing the sequences with the patients, it might possibly also have an impact on the couples feelings for the embryo and a stronger emotional bonding to a potential child. The main discrepancy is however that ultrasonography is performed in an ongoing pregnancy, whereas time-lapse is performed on the first steps of a cleaving embryo making the issue in some ways even more sensitive. All couples coming for assisted reproduction have a deep longing for a child. At the point of viewing the sequence one doesn't know if a pregnancy will be achieved. Some patients have in free comments stressed that viewing the sequence added an extra dimension to their treatment, that it 
added interesting information and that their understanding of the treatment process grew significantly. Others commented that even if they appreciated to be shown the sequence, it was not that important for them and that they just expected the clinic to use the best technique available for their treatment.

Further studies are required to analyze how much patients identify with the embryos before implantation. Concepts like identification with the embryo and the hope created by showing an embryo that is labeled "normally developed" may be studied further. That might increase the positive expectations of an ART-treatment and lead to feelings of sorrow if the treatment is unsuccessful. The results do not indicate that viewing time-lapsesequences specifically adds extra stress in this aspect. However, there have been indications outside the questionnaire of our study, that some patients who reported a negative pregnancytest also spontaneously expressed a change of view of the timelapse sequence. When expectations of the treatment were not fulfilled their feelings about the information was altered in a negative way. That cannot be analyzed in this article as we did not have the possibility to include follow up questions. It would therefore be valuable to follow up if the patient's opinions about viewing the sequence change depending on the result of the treatment. Academic literature is lacking in this area.

To view one's own diagnostic images has been shown to influence the social dynamic of a medical consultation [17]. It enhanced the patient's understanding of their medical problem and also had a potential for emotional impact (positive and negative). The study pointed out that at least for some patients the result of being shown images was that they felt more actively a part of the consultation. They felt more respected and valued by their doctor because more time was spent and because the doctor's expectation of patient's interest suggests a high opinion of the patient as a person. This effect was also seen in our study.

A possible bias could be that patients participating in the study were given extra information, and more time for information, and that that dimension per se made them more content with their treatment. To even better evaluate the role of the timelapse sequence it might be interesting to randomize and compare patient satisfaction with different set- ups of information.

Another aspect that would need further consideration is if giving away sequences could potentially "backfire" if something goes wrong later in pregnancy. Patients might compare their sequences to others or to other sources of information. Are we prepared to meet that? And can we correctly convey the message to our patients of how flimsy the correlation might be between what happens in the IVF-dish and the final outcome? In ART, traditionally there has been no possibility to get a second opinion regarding morphological evaluation of embryo quality.

The field ofART is moving forward at a fast pace and technological innovations allow progress in this field as in many others. The information on embryo development gathered through timelapse technology is certainly important and interesting. Data has been presented to support that this information can be translated into patient benefit [18]. At this time however, while the use of time-lapse technology is rapidly increasing, it is a not clear if IVF-clinics can show clear improvements in pregnancy outcomes when using this technology [19]. In our eagerness to develop our supporting technology, we must not forget the couple who are in the middle of this whirlwind of technological development.

\section{Conclusions}

The use of time-lapse must be evidence based. A medical value and the benefits for the lab-procedure and for the IVF-treatment are fundamental. If the technique is available in a clinic and used with this intention it could then be an additive value to share the sequences with the patients as deepened information about their treatment. We found that patients considered viewing the sequences to be a relevant and important aspect of their treatments. On the whole, the positive comments dominated and if the practical circumstances in a clinic allow it, it could be considered that it would be worthwhile to include this information in the treatment and that it would add extra value for the patients. As to all new routines advantages and disadvantages must be considered in prioritizing, e.g. time available for consultation at the occasion of embryo transfer and possible extra work with follow up consultations.

A majority of the participating couples expressed a clear interest in obtaining a copy of the time-lapse sequence but only if the treatment resulted in a pregnancy. It may therefore, in our opinion, be worth considering only giving a copy of the sequence to patients with an ongoing pregnancy or even wait until the time of birth.

\section{Acknowledgements}

We would like to thank the embryologists, nurses, midwives, doctors and other colleagues at the clinics who participated in the data collection for the study.

\section{References}

1. Hardarson $\mathrm{T}$, Lofman $\mathrm{C}$, Coull $\mathrm{G}$, et al. Internalization of cellular fragments in a human embryo: time-lapse recordings. Reprod Biomed. 2002;5:36-8.

2. Payne D, Flaherty SP, Barry MF, et al. Preliminary observations on polar body extrusion and pronuclear formation in human oocytes using time-lapse video cinematography. Hum Reprod. 1997;12:532-41.

3. Armstrong S, Vail A, Mastenbroek S, et al. Time-lapse in the IVF-lab: how should we assess potential benefit? Hum Reprod. 2015;30:3-8.

4. Freour T, Basile N, Barriere P, et al. Systematic review on clinical outcomes following selection of human preimplantation embryos with time-lapse monitoring. Hum Reprod Update. 2015;21:153-4.

5. Kirkegaard K, Ahlström A, Ingerslev HJ, et al. Choosing the best embryo by time lapse versus standard morphology. Fertil Steril. 2005;103:323-32.

6. Rubio I, Galán A, Larreategui Z, et al. Clinical validation of embryo culture and selection by morphokinetic analysis: a randomized, controlled trial of the EmbryoScope. Fertil Steril. 2014;102:1287-94.

7. https://www.carefertility.com/treatments/embryology-treatments/ caremaps/

8. http://www.ivi-fertility.com/en/patients/assisted-reproductiontechniques/Embryoscope/ 
Citation: Blomquis AB, Samir R, Engström AB, et al. Patients experience of viewing time-lapse sequences: A prospective survey study. Gynecol Reproduct Endocrinol -UK. 2017;1(1):1-6

9. Sandelowski M. Channel of Desire: Fetal Ultrasonography in Two Use-Contexts. Qual Health Res. 1994;4:262-80.

10. Ji EK, Pretorius DH, Newton R, et al. Effects of ultrasound on maternal-fetal bonding: a comparison of two- and three-dimensional imaging. Ultrasound Obstet. Gynecol. 2005;25:473-7.

11. Williams C, Sandall J, Lewando-Hundt G, et al. Women as moral pioneers? Experiences of first trimester antenatal screening. Soc Sci Med. 1992;61:1983-92.

12. Boivin J, Griffiths E, Venetis CA. Emotional distress in infertile women and failure of assisted reproductive technologies: meta-analysis of prospective psychosocial studies. BMJ. 2011;342:223.

13. Rooney KL, Domar AD. The impact of stress on fertility treatment. Curr Opin Obstet Gynecol. 2016;28:198-201.

14. Verhaak CM, Smeenk JM, Evers AW, et al. Women's emotional adjustment to IVF: a systematic review of 25 years of research. Hum Reprod Update. 2007;13:27-36.

15. Kupka MS, Ferraretti AP, de Mouzon J, et al. European
IVF-Monitoring Consortium, for the European Society of Human Reproduction and Embryology. Assisted reproductive technology in Europe, 2010: results generated from European registers by ESHRE. Hum Reprod. 2014;29:2099-113.

16. Krones T, Schlüter E, Neuwohner E, et al. What is the preimplantation embryo? Soc Sci Med. 2006;63:1-20.

17. Carlin LE, Smith HE, Henwood F. To see or not to see: a qualitative interview study of patients' views on their own diagnostic images. BMJ Open. 2014;4:e004999.

18. Gardner DK, Meseguer M, Rubio C, et al. Diagnosis of human preimplantation embryo viability. Hum Reprod Update. 2015;21:727-47.

19. Kieslinger DC, De Gheselle S, Lambalk CB, et al. Embryo selection using time-lapse analysis (Early Embryo Viability Assessment) in conjunction with standard morphology: a prospective two-center pilot study. Hum Reprod. 2016;31:2450-57.

\section{*Correspondence to:}

Julius Hreinsson

Department of Immunology, Genetics and Pathology Rudbeck Laboratory

Uppsala

Sweden

Tel: 023-78 3040

E-mail: julius.hreinsson@ivfkliniken.se 